\title{
Fabrication of low aspect ratio three-element Boersch phase shifters for voltage-controlled three electron beam interference
}

Cite as: J. Appl. Phys. 128, 134502 (2020); doi: 10.1063/5.0020383

Submitted: 30 June 2020 - Accepted: 14 September 2020 .

Published Online: 6 October 2020

Pooja Thakkar, ${ }^{1,2, a)}$ (ID Vitaliy A. Guzenko, ${ }^{3}$ (D) Peng-Han Lu, ${ }^{4,5}$ Rafal E. Dunin-Borkowski, ${ }^{4}$ Jan Pieter Abrahams, ${ }^{1,2,6}$ (D) and Soichiro Tsujino ${ }^{1,2, b)}$ (D)

\author{
AFFILIATIONS \\ ${ }^{1}$ Division of Biology and Chemistry, Paul Scherrer Institut, Forschungsstrasse 111, 5232 Villigen PSI, Switzerland \\ ${ }^{2}$ Swiss Nanoscience Institute, University of Basel, Klingelbergstrasse 82, 4056 Basel, Switzerland \\ ${ }^{3}$ Photon Science Division, Paul Scherrer Institut, Forschungsstrasse 111, 5232 Villigen PSI, Switzerland \\ ${ }^{4}$ Ernst Ruska-Centre for Microscopy and Spectroscopy with Electrons and Peter Grünberg Institute, Forchungszentrum Jülich, \\ Wilhelm-Johnen-Strasse, 52425 Jülich, Germany \\ ${ }^{5}$ RWTH Aachen University, Ahornstraße 55, 52074 Aachen, Germany \\ ${ }^{6}$ Biozentrum, University of Basel, Klingelbergstrasse 70, 4056 Basel, Switzerland
}

\author{
a) Author to whom correspondence should be addressed: pooja.thakkar@psi.ch

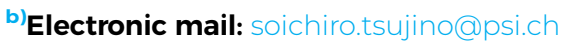

\begin{abstract}
A Boersch phase plate can shift the phase of electrons proportionally to the applied electrical potential, thereby allowing for in situ control of the electron phase shift. A device comprising multiple Boersch phase shifter elements will be able to modulate the wavefront of a coherent electron beam and control electron interference. Recently, fabrication of single and $2 \times 2$ element Boersch phase shifter devices by focused ion beam milling has been reported. Realization of a large-scale Boersch phase shifter array would demand further developments in the device design and the fabrication strategy, e.g., using lithographic processes. In the present work, we develop a fabrication method utilizing the state-of-the-art electron beam lithography and reactive ion etching processes, a combination that is widely used for high-throughput and large-scale micro- and nanofabrication of electronic and photonic devices. Using the developed method, we fabricated a three-element phase shifter device with a metal-insulator-metal structure with 100-nm-thick ring electrodes and tested its electron transmission characteristics in a transmission electron microscope with a beam energy of $200 \mathrm{keV}$. We observed voltage-controlled evolution of electron interference, demonstrating the voltage-controlled electron phase shift using the fabricated device with a phase shift of $\pi$ rad per $1 \mathrm{~V}$. We analyze the experimental results in comparison with a three-dimensional electrostatic simulation. Furthermore, we discuss the possible improvements in terms of beam deflection and crosstalk between phase shifter elements in a five-layer device structure.
\end{abstract}

Published under license by AIP Publishing. https://doi.org/10.1063/5.0020383

\section{INTRODUCTION}

Holographic synthesis of electron waves via phase front modulation has been actively studied recently. ${ }^{1-5}$ Applications of the holographic electron wavefront control include phase contrast enhancement of high-resolution microscopy, ${ }^{6-8}$ synthesis of vortex beams and higher-order Laguerre-Gaussian beams with orbital angular momentum, ${ }^{1-3,9-15}$ Bessel beams, ${ }^{16}$ Airy beams, ${ }^{17}$ and the generation of arbitrary electron wave patterns in $2 \mathrm{D}^{18}$ and $3 \mathrm{D} .{ }^{4}$
Such reported experiments demand specialized static electron phase plates that are designed via inverse modeling, fabrication, and insertion before or after the sample under investigation at a given microscope setting. The static phase plates are typically produced by nanofabrication methods such as the focused ion beam (FIB) milling ${ }^{1,2}$ or electron beam lithography. ${ }^{4}$ In contrast, a device with an active control of local phase shift, e.g., by applying voltage, will enable flexible use of such phase plates with in situ tunability 
without adapting the phase plate parameters for specific microscope settings in advance. In the case of the Boersch phase plate, ${ }^{6-8,19-23}$ it is possible to operate the device with such a flexibility. When an electron traverses through an aperture surrounded by a ring electrode, the phase of the electron is delayed or advanced by an amount proportional to the voltage applied to the electrode. Voltage-controlled phase modulating devices based on other approaches such as the nano-needle pair $^{9}$ and the nano-beam pair $^{11,15}$ have also been studied for the purpose of producing electron caustics or electron vortex beams.

An interesting and promising approach is to use an array of Boersch phase plates ${ }^{21}$ with a sufficiently large number of phase shifter elements that enables the modulation of an electron wavefront. These controllable phase plates provide greater in situ flexibility for high-resolution microscopy and spectroscopy applications without the need to exchange the static phase plates that require readjustment of observation conditions. ${ }^{21}$ Such a device may also be useful for the phasing of microcrystal electron diffractions. ${ }^{24,25}$ Analogous to the liquid crystal phase shifter array for visible light optics, ${ }^{26-29}$ an array of Boersch phase shifters will extend the possibilities of electron wavefront engineering, diffraction analysis, and spectroscopic experiments. Matsumoto and Tonomura showed that the phase shift of the Boersch phase shifter is uniform in the interior of the aperture. ${ }^{30}$ Therefore, the calculation and programming for the electron wavefront synthesis will be straightforward with such a device. In the literature, up to $10^{6}$ individually controlled electron beam arrays have been demonstrated for massively parallel electron beam lithography by incoherently splitting an electron beam, ${ }^{31-35}$ yet no such device exists for the manipulation of a coherent electron wavefront.

With a scalable and high-throughput fabrication of a largescale phase shifter array as a goal, we investigate a fabrication methodology based on electron beam lithography, ${ }^{4,13}$ which offers advantages of defining large-scale nanostructures with various materials in combination with subtractive and additive lithographic processes. ${ }^{36,37}$ In this work, we fabricate a three-element phase shifter device in a three-layer, i.e., a metal-insulator-metal (MIM) structure without a shielding electrode. The electron transmission characteristics of the phase shifter for a high-energy coherent electron beam are tested in a transmission electron microscope equipped with a field emission electron gun. The successful observation of voltage-controlled electron interference demonstrates the phase shift controllability of our device. In Sec. II, we discuss the fabrication methodology developed in this work. Section III describes the experimental procedure for testing the phase shift performance of the fabricated device. In Sec. IV, the experimental results of the voltage-controlled three-beam interference are presented along with a comparative study based on electrostatic simulations. We finally summarize and conclude the work in Sec. V.

\section{DEVICE FABRICATION PROCESSES}

Figure 1(a) shows the schematic drawings of the fabrication process steps of our device. The schematic cross section, the top-view optical image, and the top-view SEM image of the device are shown in Figs. 1(b)-1(d), respectively. The device consists of three apertures made through the insulator ( 200-nm-thick silicon nitride), which are individually surrounded by metal ring electrodes ( 100-nm-thick $\mathrm{Au})$ with a 50-nm-thick chromium (Cr) layer as the bottom electrode. The apertures and, in turn, the ring electrodes are arranged in a triangular geometry as shown in Fig. 1(d). The diameter of the apertures and the center-to-center distance between adjacent apertures measured from the SEM image are $0.90 \pm 0.05 \mu \mathrm{m}$ and $1.8 \pm 0.05 \mu \mathrm{m}$, respectively. The width of the

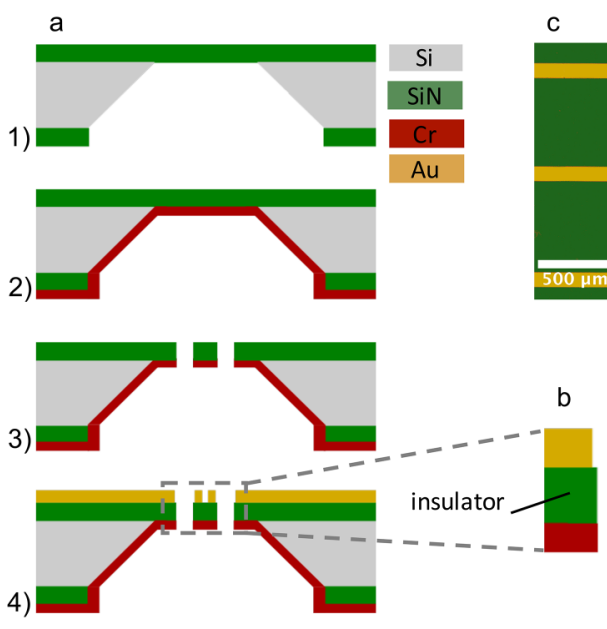

FIG. 1. (a) Schematic of fabrication process steps for Boersch phase shifter array: (1) The device is fabricated on a free-standing, low-stress SiN membrane; (2) a Cr metal layer is deposited on bottom side of the chip; (3) aperture patterning and transfer from SiN membrane to $\mathrm{Cr}$ layer; and (4) patterning of ring electrodes around etched apertures using e-beam lithography and lift-off. (b) Schematic cross section of a three-layer Boersch phase shifter device. (c) Top-view optical microscope image (false colored) of the fabricated device. Three contact strips are connected to the three phase shifter elements PE1, PE2, and PE3 fabricated within the small square depicting SiN membrane. (d) Top-view SEM image of the fabricated device, showing the three apertures with individual ring electrodes and contact strips. 
ring electrodes is $280 \pm 20 \mathrm{~nm}$, and its inner circumference is offset by $80 \pm 10 \mathrm{~nm}$ from the aperture edges. The adjacent ring electrodes are separated by $120 \pm 10 \mathrm{~nm}$. When an electron beam irradiates the device, electrons pass through the apertures. An electrical voltage $V_{\mathrm{n}}$ is applied to the ring electrode of the $\mathrm{n}^{\text {th }}$ phase element (PEn, where $n=1,2,3$ ) with respect to the bottom electrode that covers the entire bottom surface of the device chip to manipulate the phase of the electron beam.

The phase shifters are fabricated on a free-standing low-stress silicon nitride $(\mathrm{SiN})$ membrane with an area of $150 \times 150 \mu \mathrm{m}^{2}$ that is suspended on a silicon substrate. The SiN membranes are fabricated by removing the silicon substrate underneath the SiN layer [see Fig. 1(a.1), depicted as blue box in Fig. 1(c)]. To prepare the $\mathrm{SiN}$ membrane, a 200 -nm-thick SiN layer is first deposited on a 4 inch silicon wafer ( $250-\mu$ m-thick) by low pressure chemical vapor deposition on both sides of the wafer. Next, windows with an area of $\sim 500 \times 500 \mu \mathrm{m}^{2}$ are defined by photolithography and reactive ion etching (RIE) of the SiN layer on the bottom side of the wafer. Subsequently, using the un-etched SiN layer as a mask, the silicon layer is wet-etched by a $\mathrm{KOH}$ solution at $70^{\circ} \mathrm{C}$ resulting in $\sim 150 \times 150 \mu \mathrm{m}^{2}$ free-standing $\mathrm{SiN}$ membranes on the silicon wafer. In the next step [see Fig. 1(a.2)], a 50-nm-thick chromium (Cr) layer is deposited on the bottom side of the chip by electron beam evaporation that serves as the bottom electrode.

The Boersch phase shifters are fabricated on the SiN membrane using a multi-step lithographic process. In order to achieve an overlay precision of $\sim 5 \mathrm{~nm}$ between the consecutive lithographic steps, six sets of rectangular alignment markers (70-nm-thick Au) in two different sizes for coarse and fine alignments are fabricated using a combination of electron beam lithography and lift-off processes. All the subsequent lithographic steps were carried out by performing an alignment routine with respect to the corresponding set of markers to ensure an accurate translational and rotational alignment of the chip.

In the subsequent step [Fig. 1(a.3)], the apertures through both $\mathrm{SiN}$ and $\mathrm{Cr}$ layers are fabricated. We pattern the resist mask (600-nm-thick PMMA $950 \mathrm{kDa}$ ) using an electron beam lithography tool (Vistec EBPG5000 PlusES operated at $100 \mathrm{kV}$ ). The SiN membrane is then etched by RIE (Oxford PlasmaPro 100) using the patterned PMMA as an etching mask. The gas mixture of $\mathrm{CHF}_{3}(40 \mathrm{sccm})$ and $\mathrm{Ar}(25 \mathrm{sccm})$ is used to create plasma at $115 \mathrm{~W}$ RF power and $25 \mathrm{~W}$ inductively coupled plasma (ICP) power at a chamber pressure of $30 \mathrm{mTorr}$ resulting in a $\mathrm{SiN}$ etch rate of $\sim 10 \mathrm{~nm} / \mathrm{min}$. Contrary to the conventionally used $\mathrm{CHF}_{3} / \mathrm{O}_{2}$ gas mixture, $\mathrm{O}_{2}$ was replaced by $\mathrm{Ar}^{38}$ which allowed us to reduce the etch rate of PMMA to the same amount as that of SiN. This way, we could etch the SiN membrane without the need of a hard mask such as a Cr layer. To etch the underlying Cr layer, we transfer the chip to another RIE tool (Bell Plasmatechnologie $\mathrm{GmbH}$ ) and use a gas mixture of $\mathrm{Cl}_{2}(50 \mathrm{sccm})$ and $\mathrm{O}_{2}(20 \mathrm{sccm})$ that etches $\mathrm{Cr}$ at a rate of $\sim 25 \mathrm{~nm} / \mathrm{min}$.

In the final step [Fig. 1(a.4)], we fabricate the ring electrodes and their contact strips [Fig. 1(c)] by a lift-off process. The PMMA (400-nm-thick) mask is patterned by electron beam lithography. After the electron beam exposure and development of the resist, a $\sim 7$-nm-thick Cr layer is deposited as the adhesion promoting layer followed by a $100-\mathrm{nm}$-thick Au layer, both by the electron beam

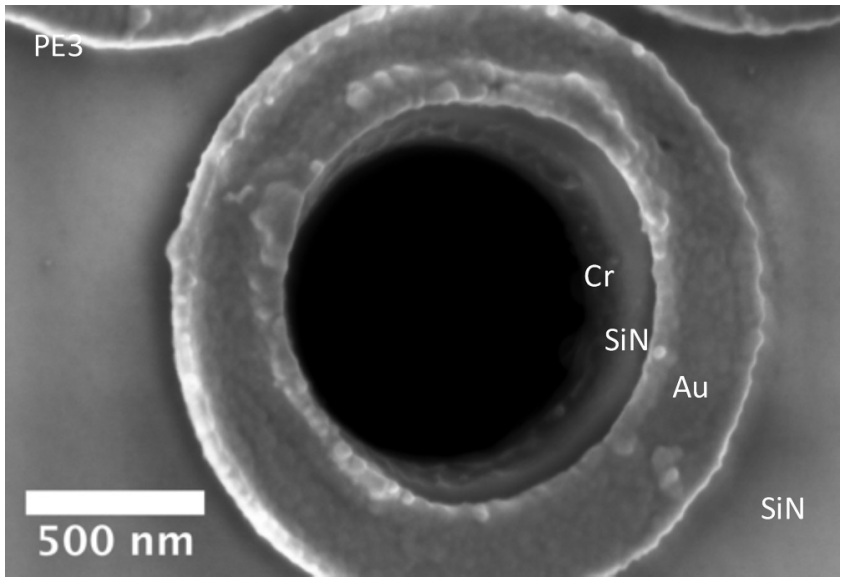

FIG. 2. High-resolution SEM image of the phase shifter element PE3 recorded at $\sim 15^{\circ}$ tilt.

evaporation method, on top of the patterned resist mask. The chip is subsequently dipped into acetone to complete the lift-off of the evaporated metal layer. An offset of $\sim 80 \mathrm{~nm}$ is created between the edge of the apertures and the inner circumference of the ring electrodes for the purpose of supporting the resist layer over the apertures. This way, the metal deposition on the aperture sidewalls was prevented and the yield of the lift-off of the deposited metal layers was improved. The dose for the resist exposure was optimized to improve the efficiency of the lift-off process by exposing the ring electrode and connecting strips inside the membrane area at 1.5 times higher dose levels than peripheral larger patterns such as contact strips and contact pads.

In Fig. 2, we show a high magnification SEM image of the phase shifter element 3 (PE3) observed at a tilt angle of $15^{\circ}$. The sharp edge of the Au ring electrode and the smooth SiN sidewall inside the aperture are visible. It can be noticed that the $\mathrm{Cr}$ electrode and the $\mathrm{Au}$ ring electrode are electrically isolated, owing to the self-aligned etching process of the SiN aperture and the $\mathrm{Cr}$ layer underneath. The electrical isolation between electrodes was confirmed in vacuum $\left(<10^{-7} \mathrm{mbar}\right)$ up to $20 \mathrm{~V}$, which is an order of magnitude higher than the amount required to shift the phase by $2 \pi$ as discussed below.

\section{MEASUREMENT OF THE PHASE SHIFTER CHARACTERISTICS}

The phase shift characteristics of the fabricated device were tested in an FEI Tecnai G2 F20 field emission transmission electron microscope equipped with a CCD camera (Gatan UltraScan $1000 \mathrm{P}$ ) with an electron beam energy of $200 \mathrm{keV}$. The device was mounted on a holder with four electrical feedthroughs (DENSsolutions SH30), which was subsequently inserted to the sample position of the microscope with the top side facing toward the electron source. A parallel electron beam was irradiated onto the sample and the transmitted electron was imaged by the detector. The holder that was in electrical and mechanical contact with 


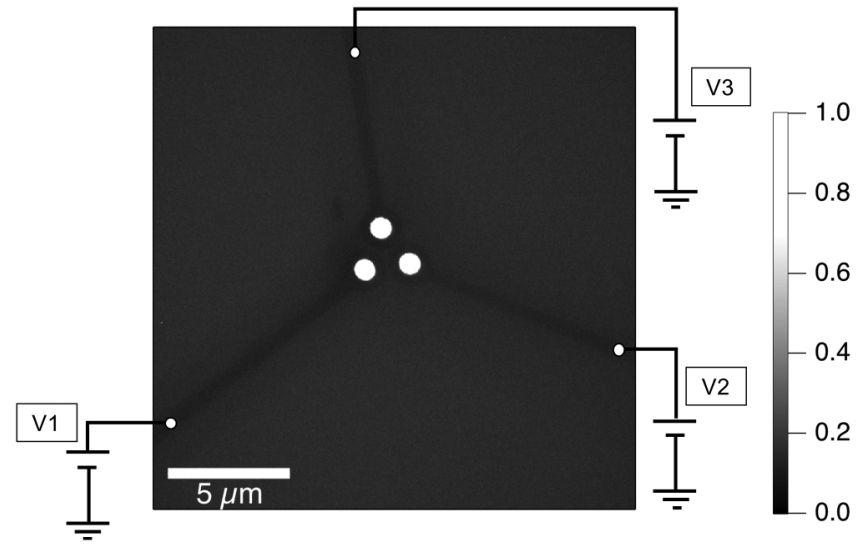

FIG. 3. A low-magnification TEM image of the three-element Boersch phase shifter device. Each phase shifter element is connected to a separate DC voltage source to apply the potential $V_{n}(n=1,2,3)$.

the bottom side of the chip was biased at $\sim 2 \mathrm{~V}$ as a protection to avoid contacting the sample holder with pole pieces. A non-zero biasing of bottom electrode does not contribute to the voltage differences between different phase elements and, hence, is unimportant to the interference of the electron beams traversing through the apertures.

A low-magnification transmission image of the device when all $V_{\mathrm{n}}$ were set to $0 \mathrm{~V}$ is shown in Fig. 3. The three bright disks indicate the electrons transmitted through the apertures unobstructed (transmission of 100\%). The dark rings and strips around the apertures show close to zero transmission through the $\mathrm{Cr} / \mathrm{Au}$ layers of the ring electrodes. The transmittance of the electron beam outside the electrode area through 200-nm-thick SiN and 50 -nm-thick Cr layers is $\sim 17 \%$.

To observe the far-field interference pattern of electron beams passing through the device, we set the microscope in the diffraction mode with a weak excitation of the objective lens ( $\sim 5 \%$ in comparison to that of $\sim 80 \%$ in imaging mode) corresponding to an equivalent optical distance of $\sim 1400 \mathrm{~m}$ between the chip and the detector.

\section{RESULTS AND DISCUSSION}

\section{A. Voltage-controlled three electron beam interference}

In Fig. 4, we show electron transmission images of the device that were acquired when the microscope was set in the far-field diffraction mode. When $V_{\mathrm{n}}(\mathrm{n}=1,2,3)=0 \mathrm{~V}$ potential was applied to the PEs, we observed a hexagonal pattern of interference of three

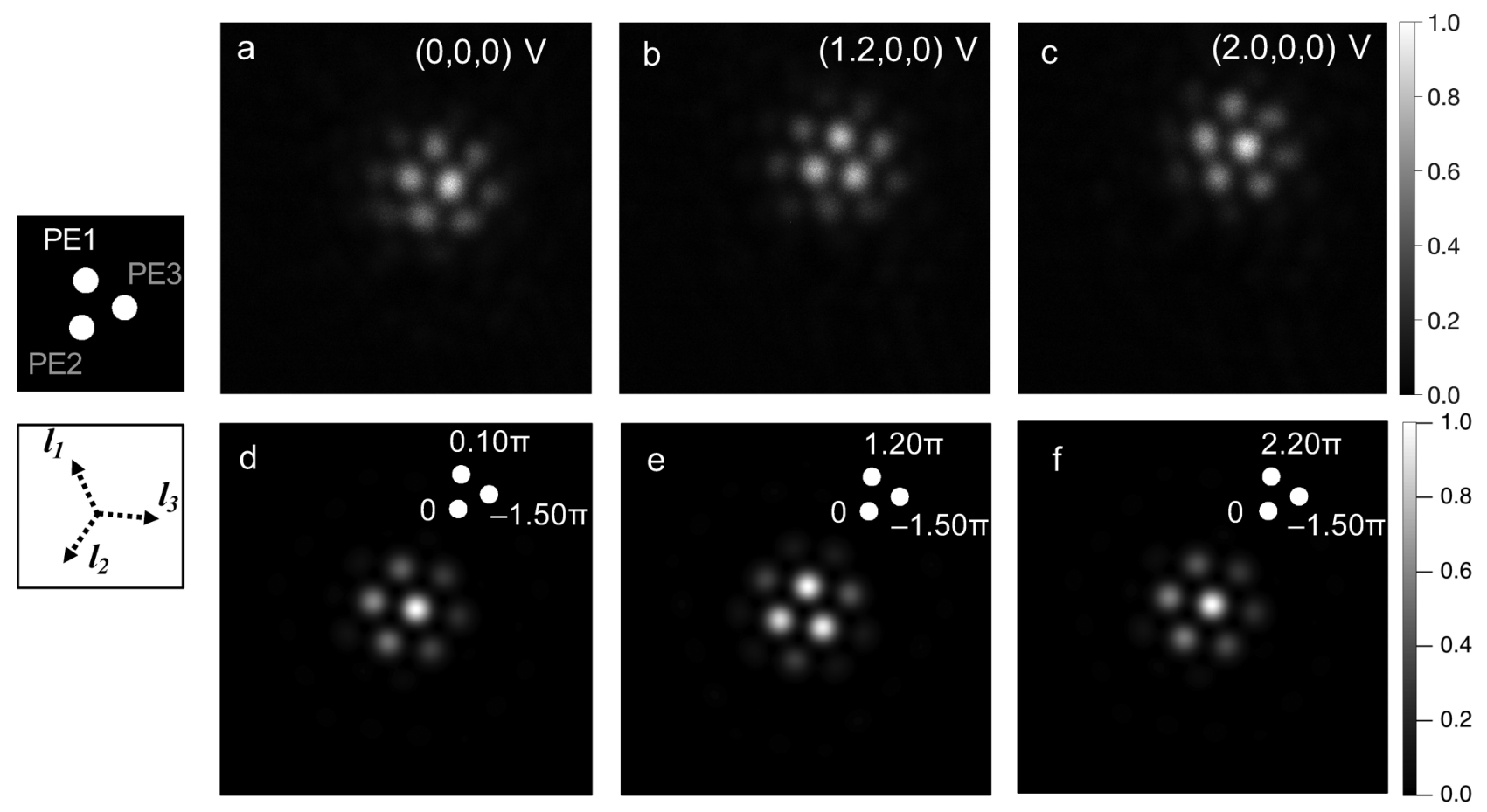

FIG. 4. Voltage-controlled three-beam interference of electrons. The transmission of a coherent $200 \mathrm{keV}$ electron beam through the fabricated three-element Boersch phase shifter device is imaged in the far-field diffraction setting. (a)-(c) show the case when the voltage $V_{1}$ applied to the phase shifter element 1 (PE1) was equal to $0 \mathrm{~V}$, $+1.2 \mathrm{~V}$, and $+2.0 \mathrm{~V}$, respectively, while voltages applied to PE2 and PE3 were equal to $0 \mathrm{~V}$. (d)-(f) show the simulated three electron beam interference with the phase of PE1 (left top aperture in the inset) was equal to $0.10 \pi, 1.20 \pi$, and $2.20 \pi$, respectively, with $-1.50 \pi$ phase offset of PE3. 
electron beams transmitted through three PEs [see Fig. 4(a) observed at $\left.V_{1}=V_{2}=V_{3}=0 \mathrm{~V}\right]$. The central interference spot is the brightest as expected from the interference of three beams with equal propagation distances. This is surrounded by hexagonally aligned spots with slightly less intensity ascribed to the first order interference spots. The observation was reproduced by Fourier transform of three-aperture image with an analogous size and geometry as our device [Fig. 4(d)]. Here, we include the phase offsets at $0 \mathrm{~V}$ indicated in the insets [Figs. 4(d)-4(f)] which were evaluated from the cross-sectional intensities. To compare the simulation with the experiment, the rotation of the three apertures is adjusted as shown in the inset in Fig. 4 [on the left top inset and in Figs. 4(d)-4(f)]. The surrounding second and higher-order diffraction spots are darker, since the finite aperture size of the PEs limits the angular spread of the transmitting electron beam within its Airy disk. The approximate cutoff of the interference pattern for the second and higher-order diffraction spots is ascribed to the fact that the ratio of the center-to-center separation of the phase elements $(\sim 1.8 \mu \mathrm{m})$ to the diameter of the aperture $(\sim 1 \mu \mathrm{m})$, approximately equal to 2 .

We next varied $V_{1}$ applied to PE1 in the range of -2 to $2 \mathrm{~V}$ while keeping $V_{2}=V_{3}=0 \mathrm{~V}$. This changed the interference pattern (see Video 1 in the supplementary material). The application of positive $V_{1}$ shifted the interference spots in the $l_{1}$-direction (see the left bottom inset in Fig. 4) toward PE1 as expected from the phase advancement of the beam through PE1 for positive $V_{1}$. When $V_{1}$ was increased from $0 \mathrm{~V}$ to $\sim 1.2 \mathrm{~V}$, the interference spots shifted until the central spot became dark. This was surrounded by two bright spots along $l_{1}$-direction which were slightly darker than the central spot at $V_{1}=0 \mathrm{~V}$. Comparing with the simulation, this is ascribed to $\sim 1.2 \pi$ phase shift of the electron beam passed through $\mathrm{PE} 1$ with respect to PE2 and PE3. Further increase of $V_{1}$ to $2 \mathrm{~V}$ continued to shift the interference spots until the image was visibly same as the case with $V_{1}=0 \mathrm{~V}$, indicating that the phase shift was approximately equal to $2 \pi$ at this voltage [see Figs. $4(\mathrm{c})$ and $4(\mathrm{f})$ ].

The same voltage-controlled variations of the interference patterns were observed while applying voltages to PE2 [see Figs. 5(a) -5 (c) and Video 1 in the supplementary material]. We also observed a similar phase shift characteristic for PE3 but with a phase offset approximately equal to $-1.5 \pi$. $V_{3}=1.2 \mathrm{~V}, 2.0 \mathrm{~V}$, and $2.8 \mathrm{~V}$ with the corresponding phase shifts approximately equal to $0, \pi$, and $2 \pi$, respectively, are shown in Figs. 5(d)-5(f). We note that the application of a small voltage as shown here could completely compensate the observed phase offset of PE3. The origin of the offset is tentatively ascribed to charging around the aperture but yet to be elucidated.

To analyze the observed voltage-controlled electron beam interference and the phase shift in detail, we extracted the intensity cross sections of the interference images along the center of the images along the $l_{\mathrm{n}}$-direction $(\mathrm{n}=1,2,3)$, shown as solid arrow in the left most insets in Figs. 4 and 5.

In Fig. 6(a), we show (in solid curves) the result of the analysis at selected voltages when $V_{1}$ was varied between 0 and $2 \mathrm{~V}$. The dashed curves show analytical simulations calculated with phase shifts that produced similar sidelobe intensity ratios (the horizontal and vertical scales of the simulated intensities were adjusted to fit with experiments). Figures 6(b) and 6(c) show the analysis of the datasets when $V_{2}$ and $V_{3}$ were varied between 0 and $2 \mathrm{~V}$ and 0 and $3 \mathrm{~V}$, respectively. The zero bias intensity profile of the $V_{1}$ dataset showed an asymmetry, suggesting a voltage offset for PE1 but with the amount much smaller than PE3 as indicated in Figs. 4 and 5.

In Fig. 7 , we summarize the relationship between the bias voltage and the phase shift for multiple $V_{\mathrm{n}}$ values evaluated from the fitting for all three PE datasets. We found that the phase shift characteristics of PE1 and PE2 are the same as indicated by the black dashed line with a slope and a phase offset of $1.03 \pi \pm 0.02 \pi \mathrm{rad} / \mathrm{V}$ and $0.10 \pi \mathrm{rad}$, respectively. The red dashed curve shows the linear fit for the case of PE3 with the phase offset of $-1.30 \pi \mathrm{rad}$. Nevertheless, the slope of $1.13 \pi \pm 0.05 \pi \mathrm{rad} / \mathrm{V}$ agrees well with the phase shift characteristics of PE1 and PE2 with an error margin of $\sim 9 \%$.

\section{B. Vortex beam generation using three phase shifter elements}

We applied voltages to three phase shifter elements equivalent to phase shifts of $-2 \pi / 3$ for PE1 and $2 \pi / 3$ for PE3 with respect to PE2. Because of the phase offset of PE3, we set $V_{3}$ equal to $1.67 \mathrm{~V}$ that approximately corresponds to the $2 \pi / 3$ phase shift of PE3 compensating the $1 \mathrm{~V}$ zero phase shift offset. This condition leads to three-beam approximation of the vortex beam with the orbital angular momentum of $1 \hbar$ [see Fig. 8(a)]. The central dark spot surrounded by three spots with the same brightness indicates the vortex singularity. The observation of Fig. 8(a) compares well with the simulation shown in Fig. 8(b). Although the three-beam approximation is rather rudimentary, the inline formation of the vortex beam instead of the finite order diffraction is potentially advantageous for applications that require controlled beam brightness and the alignment of beam on the sample.

\section{Comparison with 3D electrostatic simulation}

We compare our experimental results with an electrostatic simulation for an in-depth analysis. When an electron propagates through a Boersch phase shifter device, the phase of the electron is proportional to the DC voltage $V_{E}$ applied to the ring electrode of thickness $t_{\mathrm{E}}$. The ring electrode is sandwiched by a pair of electrodes that are kept at ground potential keeping the same electron energy of the incident and transmitted beams. ${ }^{7,20}$ Assuming that the potential inside the phase shifter element varies stepwise between 0 and $V_{\mathrm{E}}$ within the thickness $t_{\mathrm{E}}$ of the ring electrode as in the case when an electron propagates through a material with the potential of $V_{E}$, the phase shift $\Delta \phi$ is given by $\sigma V_{\mathrm{E}} t_{\mathrm{E}}$. The coefficient $\sigma$ is given by the following equation for an electron with the kinetic energy $E$ (in the order of the electron rest mass energy $E_{0}$ ), where $E \gg V_{E}:{ }^{39}$

$$
\sigma=\frac{2 \pi}{\lambda} \frac{E+E_{0}}{E\left(E+2 E_{0}\right)}
$$

In Eq. (1), $\lambda$ is the electron wavelength. For a $200 \mathrm{keV}$ electron beam $(E=200 \mathrm{keV}), \lambda=2.5 \mathrm{pm}$ and $\sigma \approx 7.29 \times 10^{6} \mathrm{rad} \mathrm{V}^{-1} \mathrm{~m}^{-1}$.

The variation of the potential along the beam trajectory in the actual device is gradual. Therefore, the maximum voltage and the effective width of the potential distribution depend on the electron 

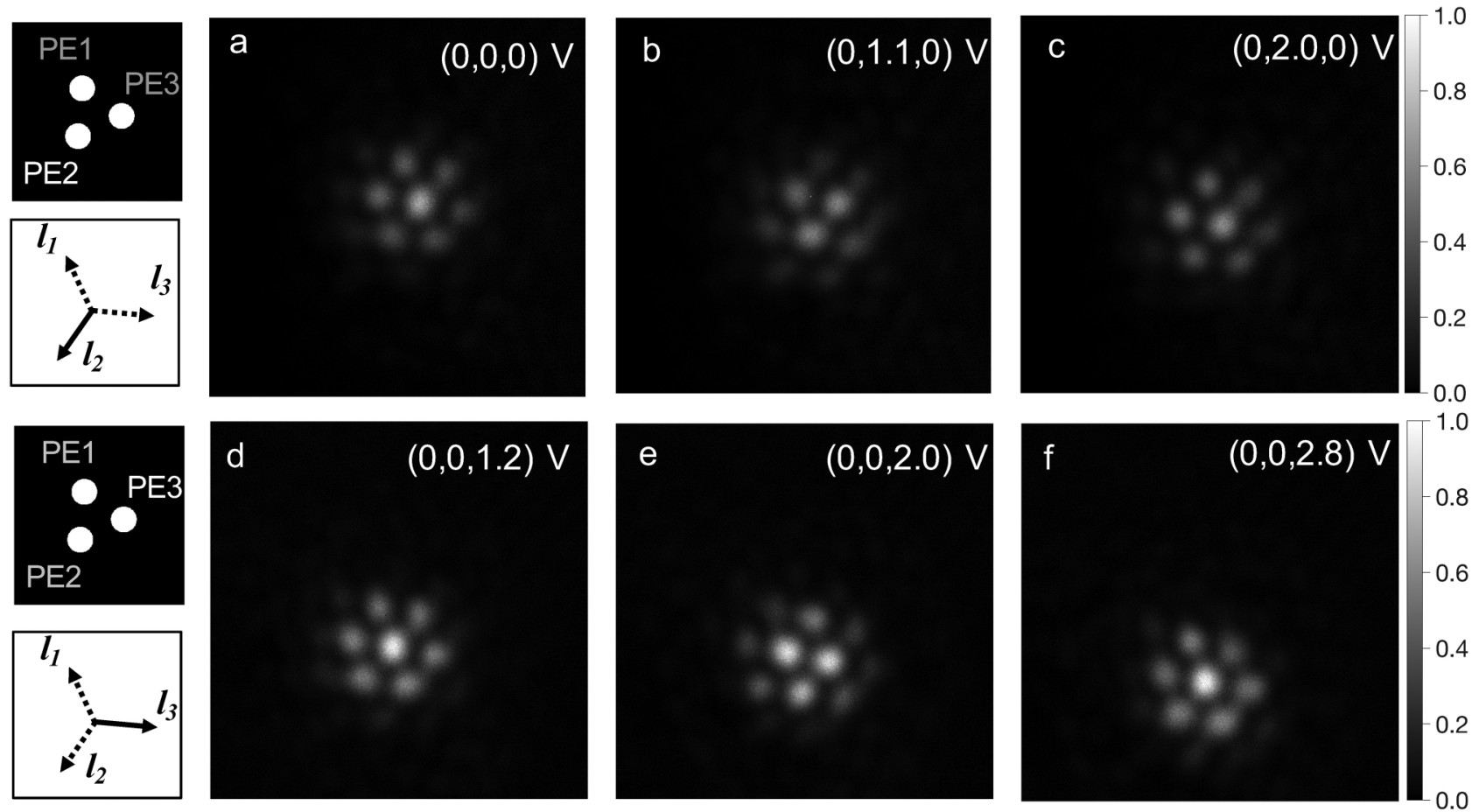

FIG. 5. Electron interference patterns for voltage variation of PE2 and PE3: for $V_{2}=0 \mathrm{~V}, 1.1 \mathrm{~V}$, and $2.0 \mathrm{~V}$ and $V_{1}=V_{3}=0 \mathrm{~V}[(\mathrm{a})-(\mathrm{c})]$ and for $V_{3}=1.2 \mathrm{~V}, 2.0 \mathrm{~V}$, and $2.8 \mathrm{~V}$ while $V_{1}=V_{2}=0 \vee[(d)-(f)]$.

trajectory subjected to the different distance from the ring electrode. However, the influence of this on phase shift $\Delta \phi$ is negligible as demonstrated by Matsumoto and Tonomura. ${ }^{30}$ The phase shift is constant in the $x-y$ plane within the aperture and is given by the following integral:

$$
\Delta \phi=\sigma \int_{-\infty}^{\infty} U(x, y, z) d z
$$

where $U(x, y, z)$ is the potential distribution produced by the phase shifter element (the $x-y$ plane is parallel and the $z$-direction is perpendicular to the sample plane and parallel to the electron trajectory). The $z$-dependence of $U(x, y, z)$ and $\Delta \phi$ deviates from the stepwise approximation of the potential variation, especially in our geometry wherein one of the electrodes sandwiching the ring electrode is omitted.

For a quantitative comparison with experiment, we calculated the three-dimensional electrostatic potential distribution of our device by a finite element solver (COMSOL Multiphysics) and evaluated $\Delta \phi$ using Eq. (2). We assumed the same size and geometry for the three phase elements as the fabricated device. Here, we discuss the results of the simulation without contact strips, and its influence is discussed in Sec. IV D.

In Fig. 9, we show the calculated potential distribution when $V_{1}=1 \mathrm{~V}$ and $V_{2}=V_{3}=0 \mathrm{~V}$. Figure 9 (a) shows the potential distribution along the plane parallel to the $x-y$ plane at $z=0.30 \mu \mathrm{m}$ (at the surface of the top electrode). The bottom electrode surface lies along the $x-y$ plane at $z=0 \mu \mathrm{m}$ and Fig. 9(b) shows the potential along the plane through the center of PE1 and PE2 [dashed line in Fig. 9(a)] parallel to the $z$ axis. Figures 9(a) and 9(b) show that the potential beneath the bottom electrode goes to zero sharply but elongated in the positive $z$-direction by $\sim 1 \mu \mathrm{m}$ above the ring electrode. Figure 9 (c) depicts the potential variation along the selected trajectories, $L_{\mathrm{c}}$ (center of PE1), $L_{\mathrm{i}}$ (at the edge of PE1 near PE2), and $L_{\mathrm{e}}$ (at the edge of PE1 away from PE2 and PE3) in PE1 parallel to the $z$ axis, marked in Fig. 9(a). For the trajectory $L_{\mathrm{c}}$, the width of the potential along the trajectory as determined by the integral in Eq. (2) is equal to $\sim 0.88 \mu \mathrm{m}$. This is factor $\sim 9$ larger than the thickness of the ring electrode $(\sim 100 \mathrm{~nm})$.

The potential along $L_{\mathrm{c}}$ decreased from $0.5 \mathrm{~V}$ at $z=0.3 \mu \mathrm{m}$ to $0.2 \mathrm{~V}$ at $z=1.3 \mu \mathrm{m}$. At the same time, the potential along the center trajectories of PE2 and PE3 increased due to the transverse spread of $V_{1}$. This induces a finite phase shift in PE2 and PE3 despite $V_{2}=V_{3}=0 \mathrm{~V}$. See Fig. 10(a) for the increased transverse spread at $z=1.3 \mu \mathrm{m}$ and Fig. 10(b) for the induced potential distribution along different trajectories of neighboring PEs. To evaluate this crosstalk effect, we calculated $\delta U_{\mathrm{nk}}$ given by the $z$-integration of the potential through the center trajectory of PEk when $1 \mathrm{~V}$ is applied to PEn along $L_{\mathrm{c}}$ by neglecting the non-uniformity of the induced potential. In this uniform approximation for a phase shift with linear relationship between phase shift in PEs and the biased voltage, the effect of crosstalk can be completely compensated by 


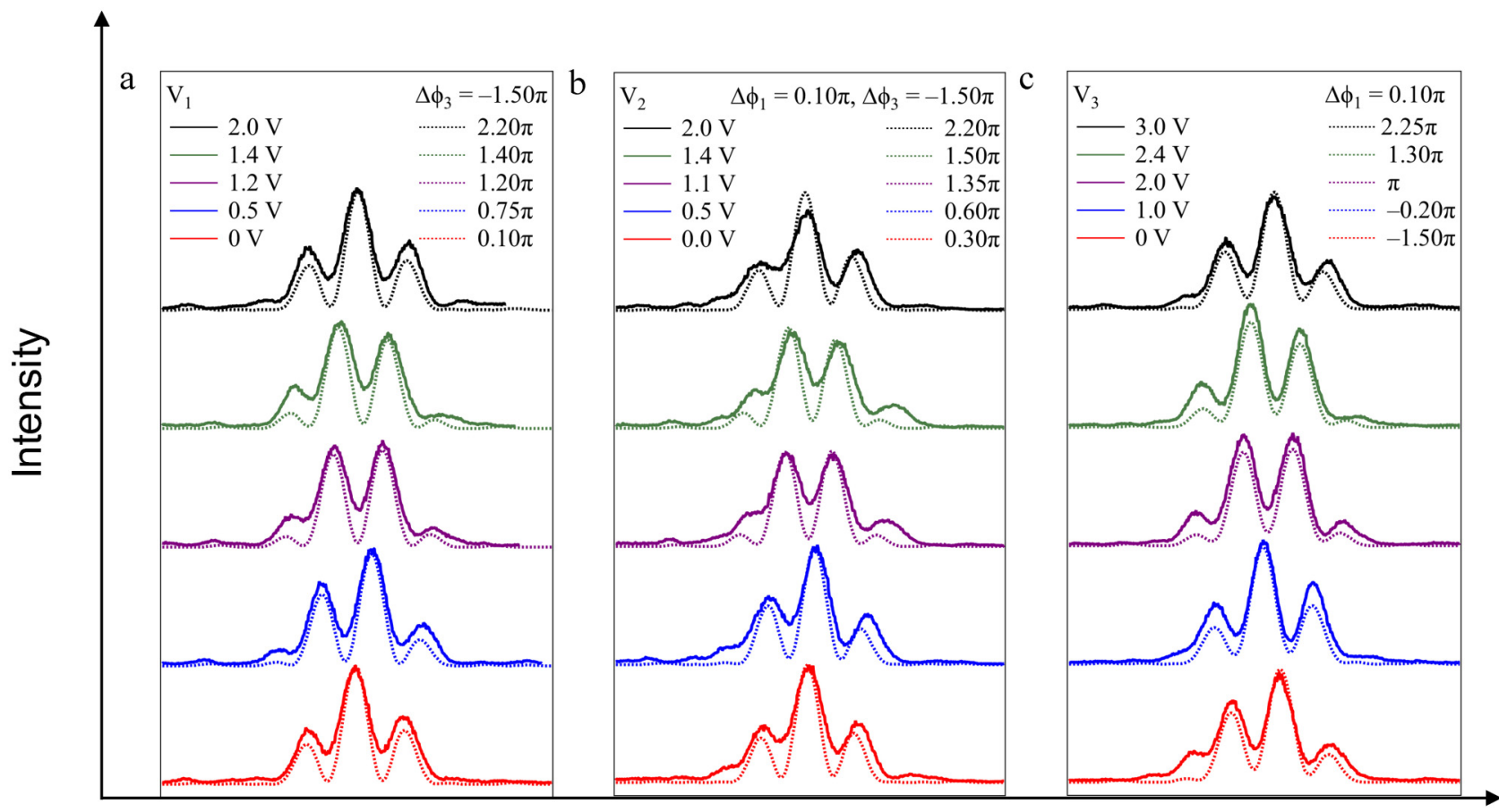

Spatial distance

FIG. 6. Intensity cross sections of the three-beam electron interference patterns evaluated from the data when $V_{1}(a), V_{2}(b)$, and $V_{3}$ (c) were varied. The cross sections are taken along (a) $I_{1}$, (b) $I_{2}$, and (c) $I_{3}$, respectively. The dotted curves show the result of fitting with theory in terms of the phase shifts of the three PEs. The fitting is conducted to the closest match for the ratio of sidelobe intensities of the experiment with the simulation.

the corresponding increase of voltage bias. The voltage $\Delta V_{1}$ required for the $\pi$ phase shift of electrons that transmit through PE1 with respect to PE2 and PE3 when $V_{1}=1 \mathrm{~V}$ and $V_{2}=V_{3}=0 \mathrm{~V}$ is given by $\pi /\left\{\sigma\left(\delta U_{11}-\delta U_{12}\right)\right\}$, which is equal to $\pi /\{\sigma$ $\left.\left(\delta U_{11}-\delta U_{13}\right)\right\}$. We found that $\delta U_{12}=\delta U_{32} \approx 0.16 \mu \mathrm{m}$. As a result, we found $\Delta V_{1}$ equal to $0.59 \mathrm{~V}$. This is $\sim 35 \%$ smaller than the experimental value. Since the larger $\Delta V_{1}$ suggests the smaller amount of $\left(\delta U_{11}-\delta U_{12}\right)$, the disagreement between theory and experiment suggests that the potential spread in the $z$-direction above the ring electrode in the experiment was shorter than the calculated value in the above simulation. Such a reduction of the potential spread might be induced by the presence of surface charge over the device surface as a result of high-energy electron beam transmission, which could result in a partial screening of the potential. We note that in our simulation, although we did not consider the difference of the surface potential (or work function) of the ring electrode and the bottom electrode explicitly. The variation of surface potential over the electrode surface due to oxidation, impurity, and residual resist are not considered. The precise effects of these parameters are unknown and require further theoretical and experimental research, which is beyond the scope of present work.

Since the transverse spread of the potential induced by PE1 is non-uniform over PE2 and PE3, the parasitic phase shifts in PE2 and PE3 are not uniform either. For our device geometry, we found from the simulation that the parasitic phase shift difference in PE2 and PE3 across aperture diameter $D=1 \mu \mathrm{m}$ (from the point nearest to PE1 to the furthest) is equal to $\sim 0.2 \pi$ at $V_{1}=1 \mathrm{~V}$ and $V_{2}=V_{3}=0 \mathrm{~V}$. The value is evaluated from the calculated potential distribution curve in the $z$-direction shown in Fig. 10(b). The first order effect of such non-uniformity is deflection of the electrons transmitting through PE2 and PE3 toward PE1. The orders of magnitude estimate of the deflection angle is given by $0.2 \pi \lambda / D$ and on the order of $\mu \mathrm{rad}$ as observed in the experiment (see Sec. IV D). This is one of the drawbacks of the MIM structure, which will be eliminated in the metal-insulator-metal-insulator-metal (MIMIM) structure as discussed in Sec. IV E.

\section{Parasitic deflection of electron beam}

In addition to the voltage-induced phase shift, we observed the deflection of the whole interference pattern during our experiment (see Figs. 3 and 4, and Video 1 and Fig. S1 in the supplementary material). The interference pattern was deflected in the $l_{\mathrm{n}}$-direction toward PEn for positive $V_{\mathrm{n}}$ and was reversed for negative $V_{\mathrm{n}}$. The amount of the deflection was approximately proportional to $V_{\mathrm{n}}$ within a factor of $\sim 2$ variation for all PEs including both polarities. Therefore, we consider that the deflection was induced by the potential gradient in the transverse direction. 


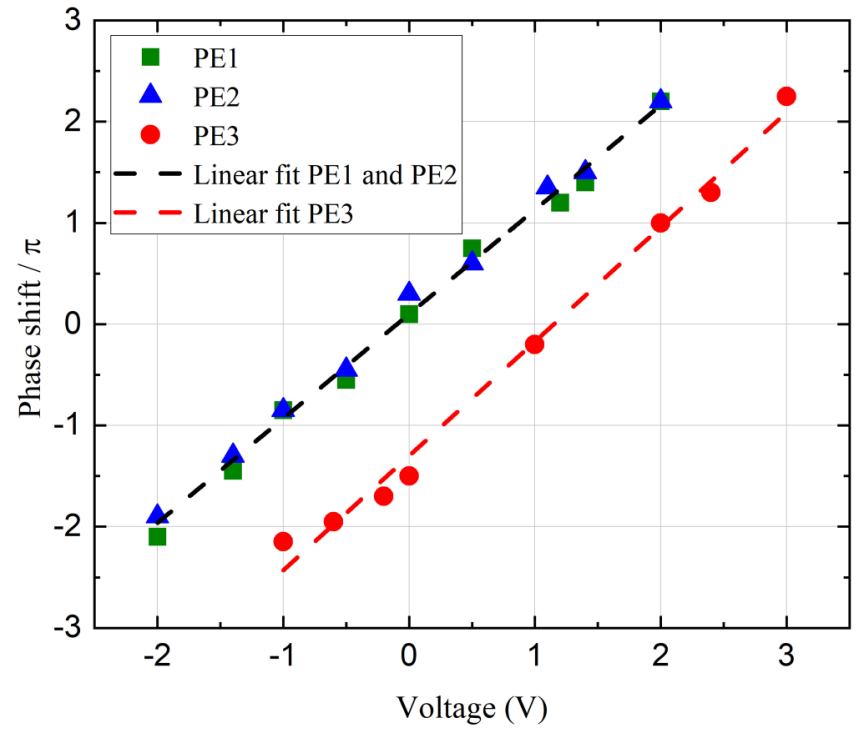

FIG. 7. The relationship between the phase shift and the bias voltage for individual PEs. The phase shift was evaluated from the intensity cross sections as shown in Fig. 6. The black dashed curve is the result of linear fit for the characteristics of PE1 and PE2 with a slope of $1.03 \pi \pm 0.02 \pi \mathrm{rad} / \mathrm{V}$ and a phase offset of $0.10 \pi \mathrm{rad}$. The red dashed curve is the result of linear fit for the characteristics of PE3 with a slope of $1.13 \pi \pm 0.05 \pi \mathrm{rad} / \mathrm{V}$ and a phase offset of $-1.30 \pi \mathrm{rad}$.

The uncovered contact strips connected to the ring electrode create asymmetry of the potential profile in the vicinity of the PE.

To quantify the deflection angle, we note that, when $V_{1}$ was increased from $0 \mathrm{~V}$ to $2 \mathrm{~V}$, the center of the beam shifted approximately by $S_{01} / 2$ on the screen, where we define $S_{01}$ equal to the distance between the central diffraction spot and the first order diffraction spot at $V_{1}=0 \mathrm{~V}$ on the screen. Considering the wavelength $\lambda=2.5 \mathrm{pm}$ for $200 \mathrm{keV}$ electron beam energy and the center-to-center distance $d=1.8 \mu \mathrm{m}$ between PEs, the angular distance corresponding to $S_{01}$ is equal to $\lambda / d \approx 1.4 \mu \mathrm{rad}$. From this, we evaluate the deflection angle, $\eta$ at $V_{1}=2 \mathrm{~V}$ to be equal to $\sim 0.7 \mu \mathrm{rad}$ (with a factor of $\sim 2$ for all cases of PE biasing).

We compare this value with the ratio $\eta_{\text {sim }}=u_{\mathrm{y}} / u_{\mathrm{z}}$, of the transverse velocity $u_{\mathrm{y}}$ to the longitudinal velocity $u_{\mathrm{z}}$ evaluated from simulation. The electrostatic potential of single PE attached with a contact strip (cross section shown in Fig. 11) is simulated for a single PE. The designed aperture has approximately the same diameter of the aperture and width of the ring electrode as the experiment, attached with a $0.3 \mu \mathrm{m}$ wide and $0.1 \mu \mathrm{m}$-thick contact strip (analogous to the fabricated device) elongated in the negative $y$-direction. From the simulated result, we evaluated the electric field $F_{\mathrm{y}}(z)$ in the $y$-direction along the electron trajectory parallel to the $z$-direction through the center of PE. Transverse velocity $u_{\mathrm{y}}$ of electrons away from the $\mathrm{PE}$ is calculated in paraxial approximation $\left(u_{\mathrm{z}} \gg u_{\mathrm{y}}\right)$ by the following integral:

$$
u_{\mathrm{y}} \approx \frac{e}{\gamma m_{0} u_{\mathrm{z}}} \int_{-\infty}^{\infty} d z F_{y}(z)
$$

where $u_{\mathrm{z}}=2.085 \times 10^{8} \mathrm{~m} / \mathrm{s}$ for electron beam energy of $200 \mathrm{keV}$, $\gamma m_{0}$ is the relativistic electron mass $(\gamma=1.4)$, and $m_{0}$ is the electron rest mass. The length $l$ of the contact strip and the width $W_{\mathrm{d}}$ of the computing domain (centered at $x=y=0 \mu \mathrm{m}$ ) was increased until the integral in Eq. (3) reached a constant value at $l=5.3 \mu \mathrm{m}$ and $W_{\mathrm{d}}=16 \mu \mathrm{m}$. We found that, when the applied voltage is equal to $2 \mathrm{~V}, u_{\mathrm{y}}=1.16 \times 10^{2} \mathrm{~m} / \mathrm{s}$, therefore predicting $\eta_{\text {sim }}$ to be equal to
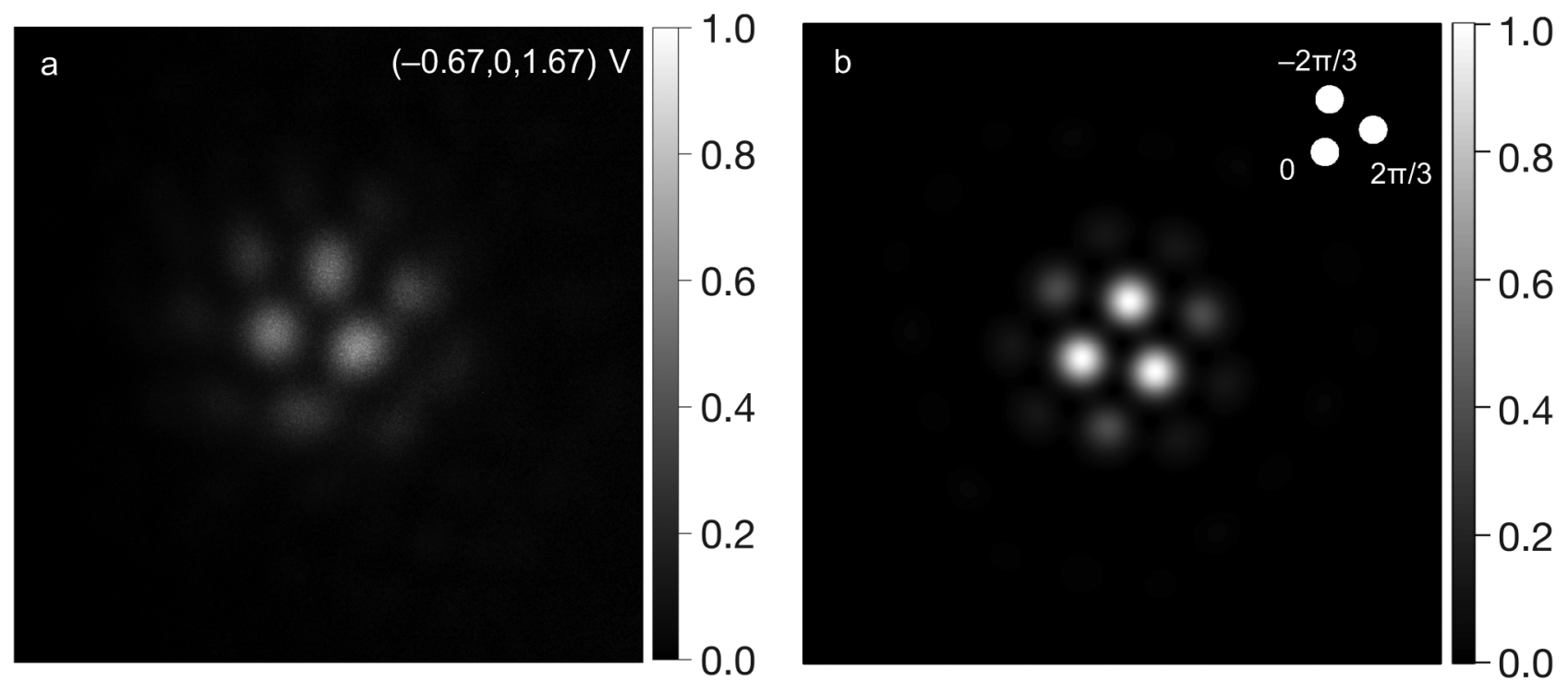

FIG. 8. (a) Electron interference pattern produced by applying the potentials $V_{1}=-0.67 \mathrm{~V}, V_{2}=0 \mathrm{~V}$, and $V_{3}=1.67 \mathrm{~V}$. The interference pattern approximates the vortex beam with the singularity at the center. (b) The corresponding simulated pattern with the phase shift equal to $-2 \pi / 3,0$, and $2 \pi / 3$ for the three PEs. 

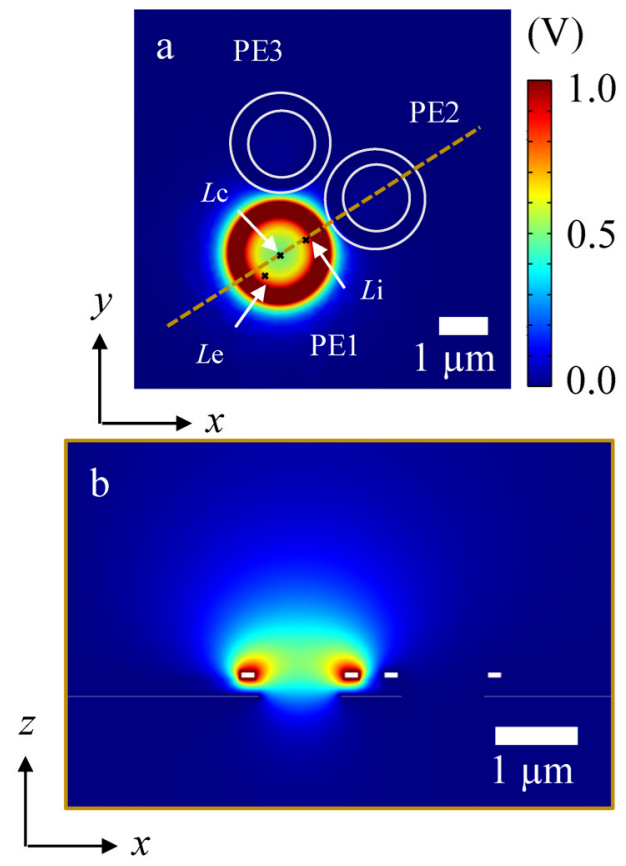

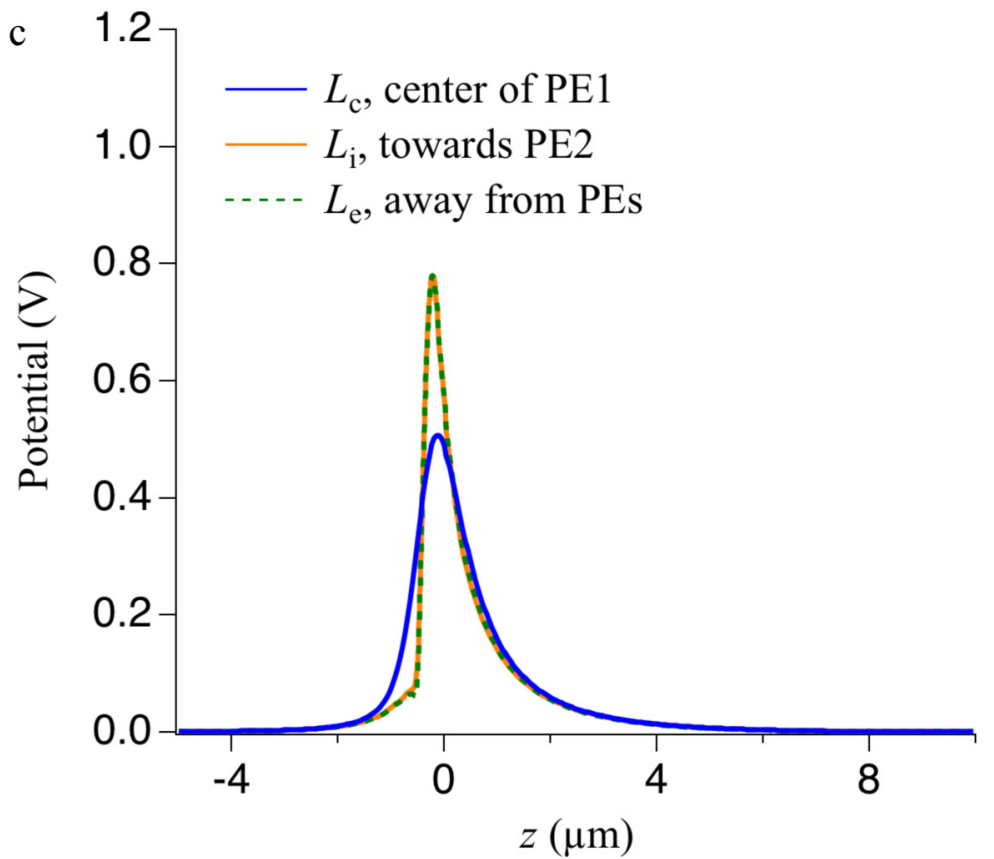

FIG. 9. The three-dimensional electrostatic potential of three-aperture phase shifter device with the metal-insulator-metal (MIM) structure, calculated at $V_{1}=1 \mathrm{~V}$ and $V_{2}=V_{3}=0 \mathrm{~V}$ by the finite element method. (a) Potential along a plane parallel to the $x-y$ plane at $z=0.30 \mu \mathrm{m}$. (b) Cross section through the center of PE1 and PE2 [along the dashed line in (a)]. (c) Variation of the electrostatic potential along the lines $L_{c}$ (center of PE1), $L_{i}$ (close to PE2), $L_{e}$ (away from PE2 and PE3) parallel to the $z$ axis, inside PE1 when $V_{2}=1 \mathrm{~V}$ and $V_{1}=V_{3}=0 \mathrm{~V}$.

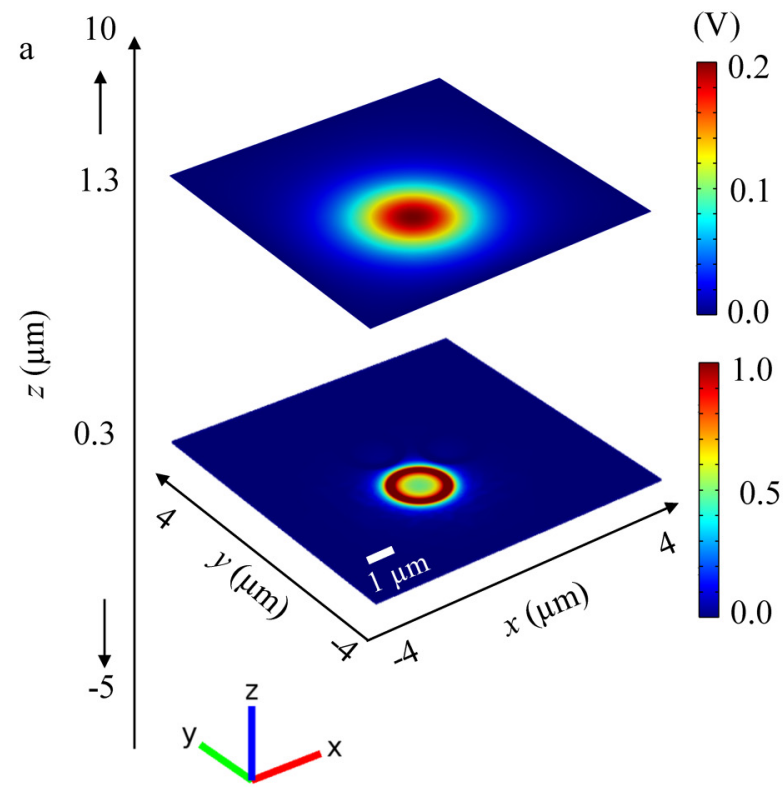

(V)

FIG. 10. (a) The calculated electrostatic potential of MIM device along the plane $z=0.3 \mu \mathrm{m}$ (just above the ring electrode) and $z=1.3 \mu \mathrm{m}(1 \mu \mathrm{m}$ above the ring electrode) for $V_{1}=1 \mathrm{~V}$ and $V_{2}=V_{3}=0 \mathrm{~V}$. (b) Variation of the electrostatic potential along the lines $L_{c}, L_{\mathrm{i}}$, and $L_{\mathrm{e}}$, within PE1 when $V_{2}=1 \mathrm{~V}$ and $V_{1}=V_{3}=0 \mathrm{~V}$. 


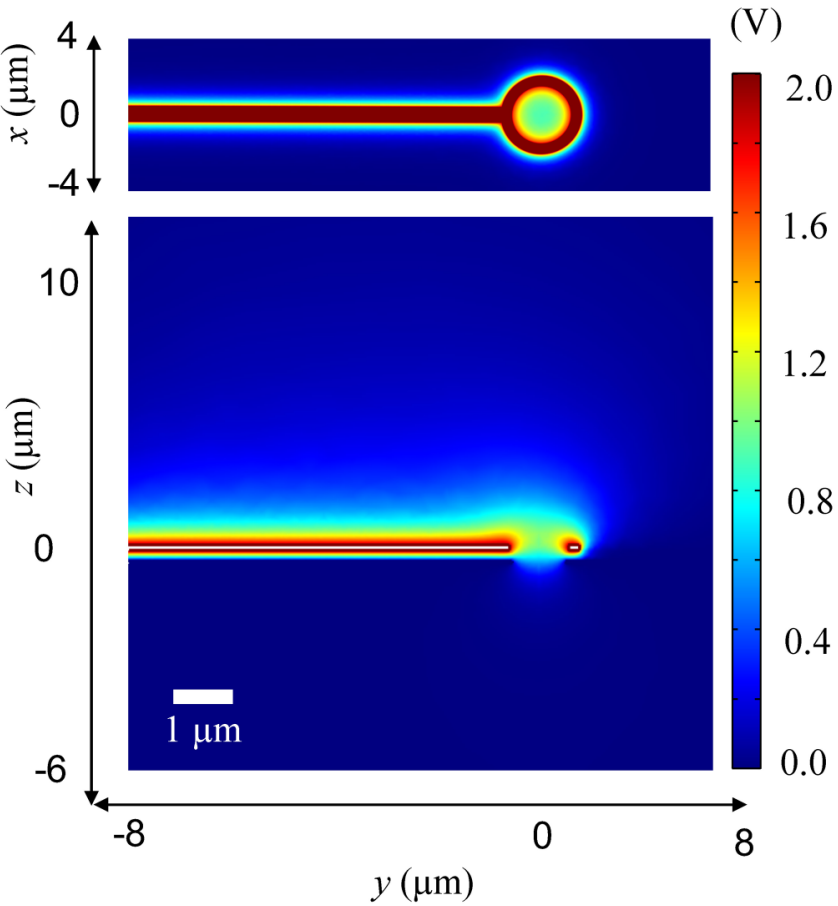

FIG. 11. 3D electrostatic potential distribution of single PE (centered at $x=y=0 \mu \mathrm{m}$ ) attached with a $0.3 \mu \mathrm{m}$ wide contact strip elongated in the $y$-direction with the same thickness as the ring electrode. The voltage of the ring electrode and the contact strip is at $2 \mathrm{~V}$ and the voltage of the bottom electrode at $0 \mathrm{~V}$. The figure shows the cross section of the potential along the plane $x=0 \mu \mathrm{m}$ passing through the center of the PE and the contact strip.

$0.56 \mu \mathrm{rad}$. This agrees well with the experiment, confirming our conclusion.

\section{E. Electrostatic simulation of the MIMIM device}

To compare the phase shift characteristics of the MIM device structure studied here with the MIMIM device structure as originally proposed by Boersch, we next calculated a three-dimensional electrostatic field of the MIMIM device. In the MIMIM structure, a second planar electrode at the same potential as the bottom electrode is added on top of the ring electrode. The device structure used in simulation is based on the structure of our MIM device, to which a $0.2 \mu \mathrm{m}$-thick second insulator and a $0.1 \mu \mathrm{m}$-thick planar electrode are added on top to the model built for the MIM device. The result calculated at $V_{1}=1 \mathrm{~V}$ and $V_{2}=V_{3}=0 \mathrm{~V}$ is shown in Fig. 12. As expected, the potential spread in the positive $z$-direction above the device is significantly reduced owing to the added planar electrode on the top at ground potential. The potential spread along the electron trajectories at the middle and near the edges of the aperture is reduced by nearly a factor of 2 . This, in turn, also increases the bias voltage by approximately a factor of 2 compared to the MIM device to achieve the same phase shift. From Eq. (2), we found that at the same applied bias, the phase shift of MIMIM device is a factor of $\sim 2.2$ smaller than the MIM device discussed in Sec. IV C.

In Fig. 13, we show the transverse spread of the potential that affected the crosstalk and its non-uniformity on the phase shift in the case of the MIM structure. In comparison to Fig. 10, the transverse spread of the potential in the MIMIM device and its influence on neighboring PEs was reduced by more than a factor of 10 . Furthermore, by shielding the contact strips of the ring electrodes by the top added electrode, its effect on the parasitic beam deflection discussed in Sec. IV D should be eliminated. These are important advantages for a device that integrates a large number of phase shifters.

\section{F. Discussion}

We evaluated visibility $v$ defined by $\left(I_{\max }-I_{\min }\right) /\left(I_{\max }+I_{\min }\right)$ of the interference ${ }^{40}$ from the zero bias intensity profile for the $V_{1}$ dataset in Fig. 6(a), where $I_{\max }$ is the average intensity of the first order maxima and $I_{\min }$ is the average intensity of the first order minima. We found the visibility values for the experiment and simulation $v_{\exp }=0.49$ and $v_{\text {sim }}=0.54$, respectively. There are several possibilities for $10 \%$ smaller visibility in experimental value such as the longitudinal and transverse coherence length of electrodes, inelastic scattering, as well as external reasons such as the microscope setting.

We consider that this is not due to an insufficient coherence length of the electron beam. The longitudinal coherence length as determined by the expected energy spread $\Delta E \approx 0.5 \mathrm{eV}$ of the electron beam emitted from the Schottky emitter is estimated by $\lambda E_{0} /(2 \Delta E) \approx 2 \times 10^{5} \lambda$ equal to $\sim 0.5 \mu \mathrm{m} \quad(\lambda \approx 2.5 \mathrm{pm}$ for electrons with the energy of $200 \mathrm{keV}){ }^{41}$ This is sufficiently larger than the phase difference of first or second order interference peaks with the path difference of $\lambda$ or $2 \lambda$. The transverse coherence length of the incident electron beam is more likely to be a limiting factor of the visibility. However, for a quantitative comparison, it is desirable to conduct a separate but simultaneous measurement of the electron beam coherence in future experiments including the condenser aperture size dependence or measurement in a microscope with higher beam energy.

The inelastic scattering of the electron beam propagating through the apertures of the phase elements is also accountable for loss in visibility, since inelastically scattered electrons would still reach the detector within the Airy disk radius (within the first order interference) but as a background, thereby increasing the intensity at the troughs. We also note that the relative intensity of the second order interference spots with respect to the zeroth and first order interference spots is several times larger in the experiment than in the simulation. In the latter, the sharp cutoff of the intensity beyond the angles determined by the aperture diameter makes the second spots extremely dark as the intensity profile of the simulated interference pattern in Fig. 6 shows. We found that this is difficult to ascribe to the smaller aperture diameter or larger center-to-center distance than those estimated from SEM. This can partly be attributed to the non-zero angular divergence of the incident beam, even though the incident electron beam was nominally aligned as parallel and vertical on the device chip. Further optimization of the interference visibility and elucidation of the visibility 

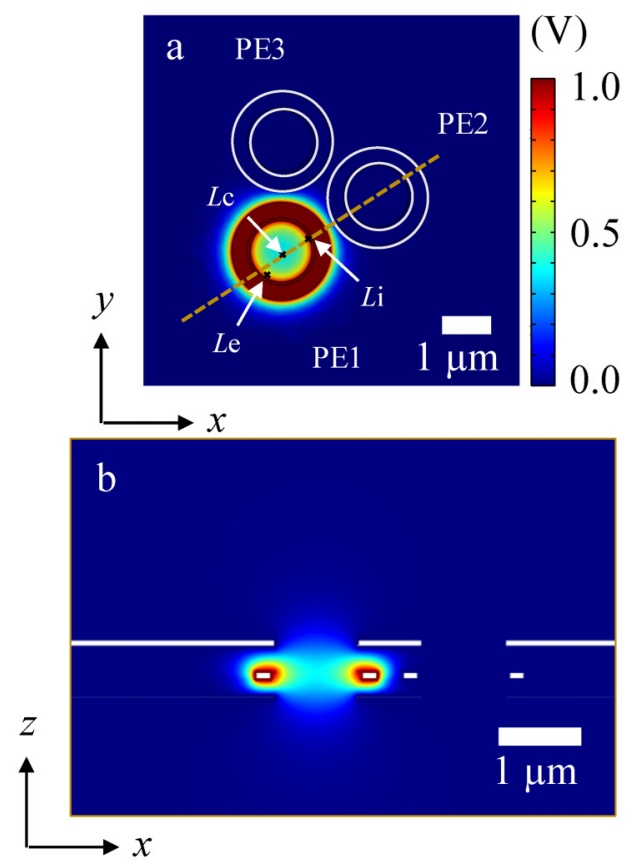

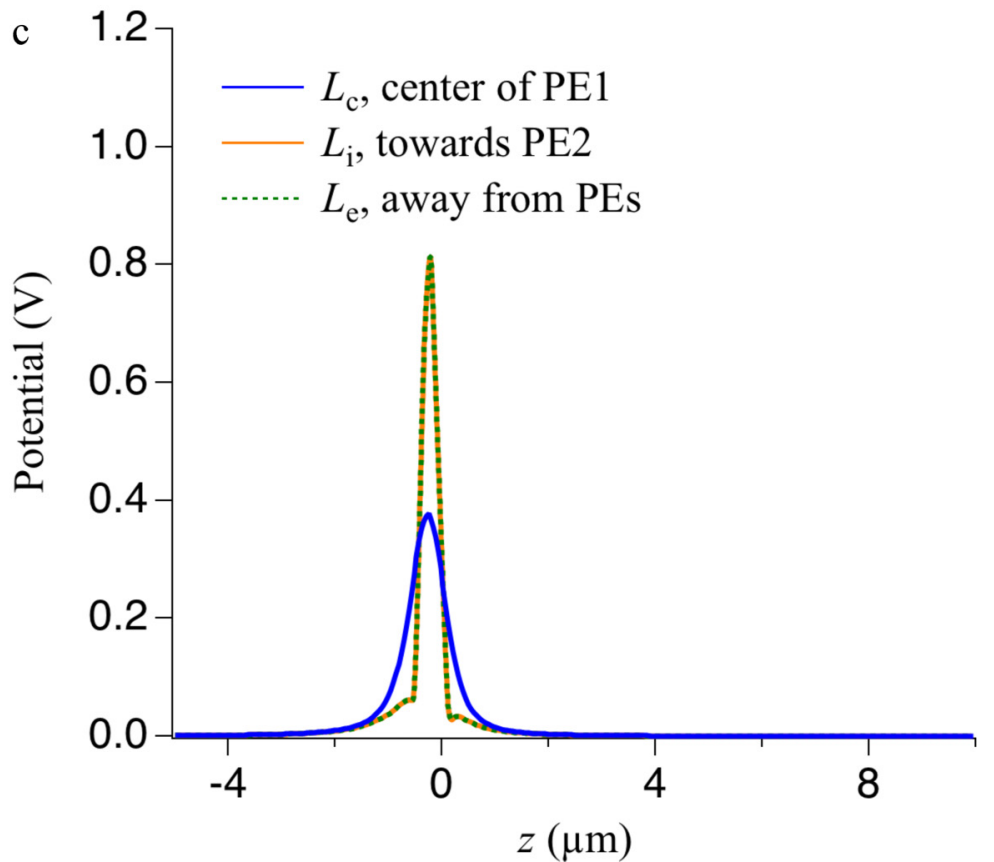

FIG. 12. A $3 \mathrm{D}$ electrostatic potential of the three-aperture phase shifter device with metal-insulator-metal-insulator-metal (MIMIM) structure, calculated at $V_{1}=1 \mathrm{~V}$ and $V_{2}=V_{3}=0 \mathrm{~V}$ by the finite element method. (a) The potential along the plane $z=0.3 \mu \mathrm{m}$ (at top surface of the ring electrode) and (b) cross-sectional view of the potential spread through the center of PE1 and PE2 [dashed line in (a)] parallel to the $z$ axis. (c) Variation of the electrostatic potential along the lines parallel to the $z$ axis, at $L_{c}$ (center of PE1), $L_{i}$ (close to PE2), and $L_{e}$ (away from PE2 and PE3) inside PE1.
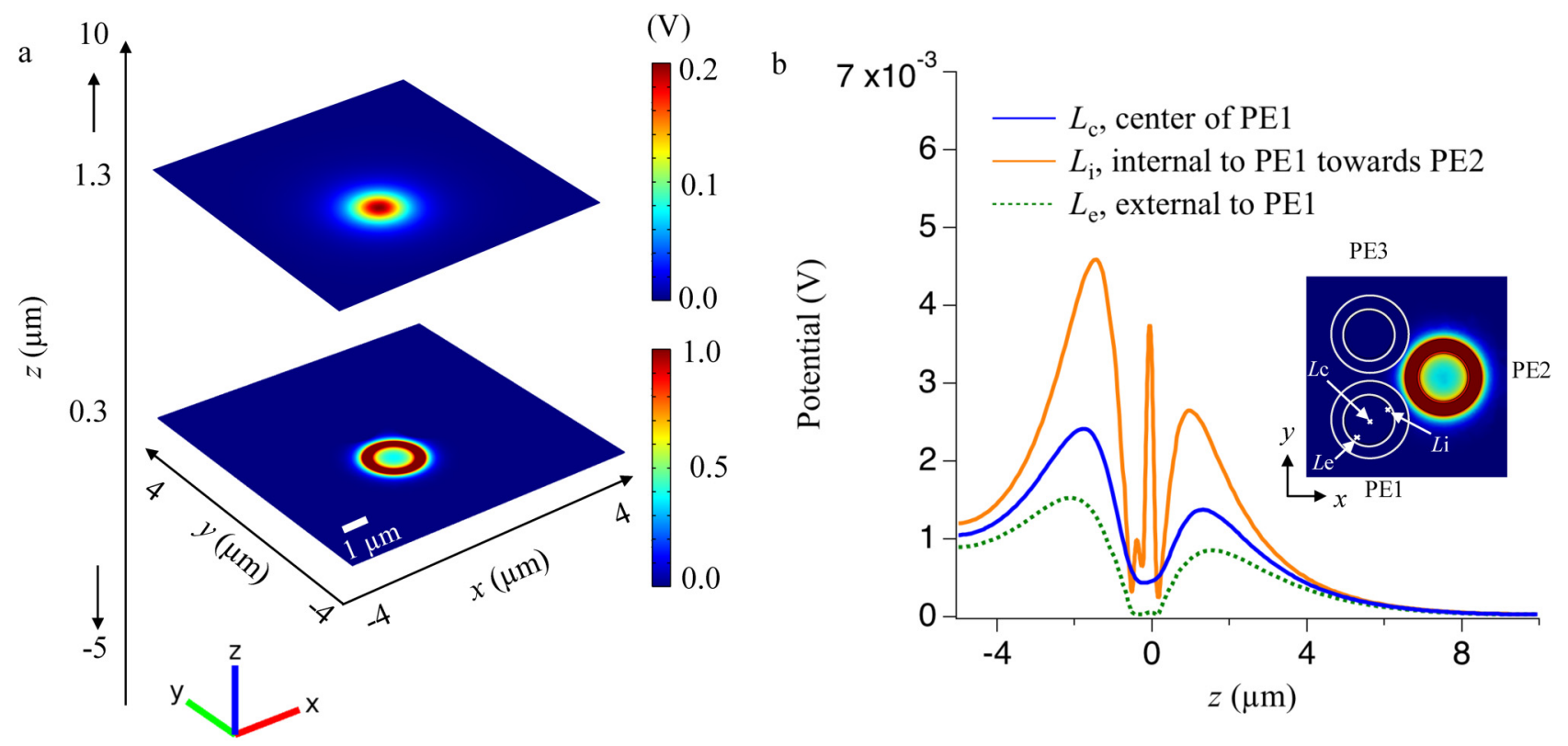

FIG. 13. (a) The calculated 3D electrostatic potential of the MIMIM device along the plane $z=0.3 \mu \mathrm{m}$ (just above the ring electrode) and $z=1.3 \mu \mathrm{m}(1 \mu \mathrm{m}$ above the ring electrode) at $V_{1}=1 \mathrm{~V}$ and $V_{2}=V_{3}=0 \mathrm{~V}$. (b) Variation of the electrostatic potential along the lines parallel to the $z$ axis at $L_{c}, L_{i}$, and $L_{e}$ inside $P E 1$. 
limiting mechanism will be mandatory for establishing the feasibility of the large PE array devices. In experiments with microscope alignment conditions such as different source aperture sizes and condenser lens adjustments, the in situ evaluation of the transverse electron beam coherence length or with the combination of the energy filtering ${ }^{42}$ would be required.

The visibility can also be reduced when the energy of the electron beams that transmitted through different apertures are slightly different: the energy difference should be within $\sim 10^{-15} \mathrm{eV}$ when the integration time of the image observation ${ }^{41,43,44}$ is in the order of $1 \mathrm{~s}$. This is particularly the case when the phase shifter voltage is non-zero. In our MIM structure without the top shielding electrode, the potential decays slowly above the biased PEs as shown in the simulation in Figs. 9 and 10. Therefore, the experimental observation of the three-beam interference, especially for $V_{\mathrm{n}}$ equal to $\sim 2 \mathrm{~V}$, and the fact that it is approximately identical to the case when $V_{\mathrm{n}}$ is equal to $\sim 0 \mathrm{~V}$, shows that the electron energies of three beams converged to a same value within $\sim 10^{-15} \mathrm{eV}$ when they propagated from the phase shifter device along the microscope column and reached to the detector. In contrast, as the simulation result shown in Fig. 11 indicates, for the case of MIMIM device structure, the convergence of the electron beam energies within the small amount will be achieved within a few micrometers propagation distance from the chip surface. This will be advantageous for applications as it will allow for placing a sample closer to the device without being affected by the beam energy difference upon incidence on the sample. This will be another potential advantage of MIMIM device structures.

Further reduction of lateral sizes with similar thickness for the same phase shift per volt efficiency ${ }^{30}$ is feasible with the developed fabrication method, which is advantageous for increased number of phase shifter elements. We would like to note that, inelastic scattering between the electron beam and electrons in the metal electrodes would comparatively increase in such reduced-lateral-size phase shifters due to shorter distances, which may subsequently diminish the visibility of interference. Therefore, from the application point of view, it will be important to find the trade-off between the number of phase shifter elements and possible loss of the beam coherence. Further quantitative study on the impact of phase shifter element size on the feasibility of fabrication including individual electrical contacts to the phase shifter elements, the phase shifter performance, and the decoherence are subjects of future study and beyond the scope of the present article.

\section{SUMMARY AND CONCLUSION}

Motivated by the exciting prospects for novel research in the field of molecular biology and materials science for developing a next generation holographic device for electron wavefront control with increased flexibility and reproducibility, we have fabricated a three-element phase shifter device using electron beam lithography and reactive ion etching, a combination that is compatible with scalable and high-throughput device fabrication. The phase shift performance of the fabricated phase shifter device was tested in a $200 \mathrm{keV}$ TEM, demonstrating an in situ voltage-controlled threebeam interference, including a rudimentary realization of a vortex beam. The analysis of the phase shift performance by way of comparing the intensity profiles with simulation showed that the phase shifter elements were capable of inducing $\pi$ phase shift with application of $\sim 0.9 \mathrm{~V}$. The phase shift per unit voltage was found to be the same for all three PEs within a margin of $9 \%$. We also observed phase offsets that could be compensated by adjusting the bias voltages with, at most, $\sim 1 \mathrm{~V}$. In comparison to the MIMIM device structure with two planar equipotential electrodes sandwiching the ring electrode surrounding the aperture, our MIM device without the top planar electrode was relatively modest to fabricate. It resulted in an effective electrode thickness of $\sim 0.6 \mu \mathrm{m}$ despite the fact that the ring electrode thickness was $0.1 \mu \mathrm{m}$ with low aspect ratio and the total thickness-to-aperture diameter ratio of $\sim 0.3$. As a potential disadvantage of the MIM device, a deflection of the beams was observed by the transverse potential gradient created by the unshielded contact strips. Also, simulation predicted a non-uniform induced phase shift of up to $20 \%$ in the neighboring apertures.

The simulation of the MIMIM device structure and its comparison with the MIM structure indicated important advantages of the former, especially for a phase shifter array device with a large number of PE elements. Therefore, together with the development of the strategy for contacting individual ring electrodes with external voltage supply, experimental validation of these advantages of MIMIM structure is an important next step of the research. Although this will increase the required voltage by nearly twice the value to achieve the same amount of phase shift, it is much lower than the dielectric breakdown limit experimentally tested for our device with the insulator thickness of $\sim 0.2 \mu \mathrm{m}$. Alternatively, the ring electrode thickness can be increased with potentially reduced device stability due to the increased stress for a device fabricated on the free-standing membrane.

\section{SUPPLEMENTARY MATERIAL}

See the supplementary material (Video 1 and Fig. S1) for a complete voltage-controlled phase shift performance of three phase shifters and the evaluation of the parasitic electron beam deflection.

\section{ACKNOWLEDGMENTS}

This work was partially funded by the Swiss Nanoscience Institute, University Basel (Project No. P1505). P.H.-L. and R.E.D.-B. acknowledge funding from the European Union's Horizon 2020 Research and Innovation Programme under Grant Agreement No. 766970 (Q-SORT). We would like to thank Professor Giulio Pozzi (University of Bologna, Italy) for his valuable insights and motivation for this work. We acknowledge Laboratory of Micro- and Nanotechnology (LMN), Paul Scherer Institute, for supporting the device fabrication. We would also like to extend our thanks to Jana Lehmann (LMN-PSI, Switzerland) for her kind help with the preparation of silicon nitride membranes.

\section{DATA AVAILABILITY}

The data that support the findings of this study are available from the corresponding author upon reasonable request. 


\section{REFERENCES}

1J. Verbeeck, H. Tian, and P. Schattschneider, Nature 467, 301 (2010).

${ }^{2}$ B. J. McMorran, A. Agrawal, I. M. Anderson, A. A. Herzing, H. J. Lezec, J. J. McClelland, and J. Unguris, Science 331, 192 (2011).

${ }^{3}$ G. Guzzinati, L. Clark, A. Béché, R. Juchtmans, R. Van Boxem, M. Mazilu, and J. Verbeeck, Ultramicroscopy 151, 85 (2015).

${ }^{4}$ R. Shiloh and A. Arie, Ultramicroscopy 177, 30 (2017)

${ }^{5}$ E. Mafakheri, A. H. Tavabi, P.-H. Lu, R. Balboni, F. Venturi, C. Menozzi, G. C. Gazzadi, S. Frabboni, A. Sit, R. E. Dunin-Borkowski, E. Karimi, and V. Grillo, Appl. Phys. Lett. 110, 93113 (2017).

${ }^{6}$ R. Cambie, K. H. Downing, D. Typke, R. M. Glaeser, and J. Jin, Ultramicroscopy 107, 329 (2007).

${ }^{7_{K}}$. Schultheiss, F. Perez-Willard, B. Barton, D. Gerthsen, and R. R. Schröder, Rev. Sci. Instrum. 77, 33701 (2006).

${ }^{8}$ J. Shiue, C.-S. Chang, S.-H. Huang, C.-H. Hsu, J.-S. Tsai, W.-H. Chang, Y.-M. Wu, Y.-C. Lin, P.-C. Kuo, Y.-S. Huang, Y. Hwu, J.-J. Kai, F.-G. Tseng, and F.-R. Chen, J. Electron Microsc. 58, 137 (2009).

${ }^{9}$ A. H. Tavabi, V. Migunov, C. Dwyer, R. E. Dunin-Borkowski, and G. Pozzi, Ultramicroscopy 157, 57 (2015).

${ }^{10}$ S.-C. Chu, Y.-T. Chen, K.-F. Tsai, and K. Otsuka, Opt. Express 20, 7128 (2012).

${ }^{1}$ G. Pozzi, P.-H. Lu, A. H. Tavabi, M. Duchamp, and R. E. Dunin-Borkowski, Ultramicroscopy 181, 191 (2017).

${ }^{12}$ V. Grillo, G. Carlo Gazzadi, E. Karimi, E. Mafakheri, R. W. Boyd, and S. Frabboni, Appl. Phys. Lett. 104, 43109 (2014).

${ }^{13}$ V. Grillo, G. C. Gazzadi, E. Mafakheri, S. Frabboni, E. Karimi, and R. W. Boyd, Phys. Rev. Lett. 114, 34801 (2015).

${ }^{14} \mathrm{M}$. Uchida and A. Tonomura, Nature 464, 737 (2010).

${ }^{15}$ A. H. Tavabi, H. Larocque, P.-H. Lu, M. Duchamp, V. Grillo, E. Karimi, R. E. Dunin-Borkowski, and G. Pozzi, Phys. Rev. Res. 2, 13185 (2020).

${ }^{16}$ V. Grillo, E. Karimi, G. C. Gazzadi, S. Frabboni, M. R. Dennis, and R. W. Boyd, Phys. Rev. X 4, 11013 (2014).

${ }^{17}$ N. Voloch-Bloch, Y. Lereah, Y. Lilach, A. Gover, and A. Arie, Nature 494, 331 (2013).

${ }^{18}$ R. Shiloh, Y. Lereah, Y. Lilach, and A. Arie, Ultramicroscopy 144, 26 (2014).

${ }^{19}$ H. Boersch, Z. Naturforsch. A 2, 615 (1947).

${ }^{20}$ E. Majorovits, B. Barton, K. Schultheiß, F. Pérez-Willard, D. Gerthsen, and R. R. Schröder, Ultramicroscopy 107, 213 (2007).
${ }^{\mathbf{2 1}}$ J. Verbeeck, A. Béché, K. Müller-Caspary, G. Guzzinati, M. A. Luong, and M. Den Hertog, Ultramicroscopy 190, 58 (2018).

${ }^{22}$ A. Walter, S. Steltenkamp, S. Schmitz, P. Holik, E. Pakanavicius, R. Sachser, M. Huth, D. Rhinow, and W. Kühlbrandt, Ultramicroscopy 153, 22 (2015).

${ }^{\mathbf{2 3}}$ A. Walter, H. Muzik, H. Vieker, A. Turchanin, A. Beyer, A. Gölzhäuser, M. Lacher, S. Steltenkamp, S. Schmitz, P. Holik, W. Kühlbrandt, and D. Rhinow, Ultramicroscopy 116, 62 (2012).

${ }^{24}$ J. M. Cowley and J. C. H. Spence, Ultramicroscopy 6, 359 (1981).

${ }^{25}$ M. Gemmi, E. Mugnaioli, T. E. Gorelik, U. Kolb, L. Palatinus, P. Boullay, S. Hovmöller, and J. P. Abrahams, ACS Cent. Sci. 5, 1315 (2019).

${ }^{\mathbf{2 6}}$ C. Slinger, C. Cameron, and M. Stanley, Computer 38, 46 (2005).

${ }^{27}$ Y. Igasaki, F. Li, N. Yoshida, H. Toyoda, T. Inoue, N. Mukohzaka, Y. Kobayashi, and T. Hara, Opt. Rev. 6, 339 (1999).

${ }^{28}$ T. Latychevskaia and H.-W. Fink, Sci. Rep. 6, 26312 (2016).

${ }^{29}$ N. Chattrapiban, E. A. Rogers, D. Cofield, I. I. I. Wendell, T. Hill, and R. Roy, Opt. Lett. 28, 2183 (2003).

${ }^{30} \mathrm{~T}$. Matsumoto and A. Tonomura, Ultramicroscopy 63, 5 (1996)

${ }^{31}$ Y. Zhang and P. Kruit, J. Vac. Sci. Technol. B 25, 2239 (2007).

${ }^{32}$ T. H. P. Chang, D. P. Kern, and L. P. Muray, J. Vac. Sci. Technol. B 10, 2743 (1992).

${ }^{33}$ P. Kruit, Microelectron. Eng. 84, 1027 (2007).

${ }^{34}$ M. Esashi, A. Kojima, N. Ikegami, H. Miyaguchi, and N. Koshida, Microsyst. Nanoeng. 1, 15029 (2015).

${ }^{35} \mathrm{C}$. Klein, H. Loeschner, and E. Platzgummer, J. Micro/Nanolithography MEMS MOEMS 11, 031402 (2012).

${ }^{36}$ M. Ekberg, M. Larsson, S. Hård, and B. Nilsson, Opt. Lett. 15, 568 (1990)

${ }^{37}$ V. A. Guzenko, A. Mustonen, P. Helfenstein, E. Kirk, and S. Tsujino, Microelectron. Eng. 111, 114 (2013).

${ }^{38}$ H. K. Lee, K. S. Chung, and J. S. Yu, J. Korean Phys. Soc. 54, 1816 (2009).

${ }^{39}$ L. Reimer, Transmission Electron Microscopy: Physics of Image Formation and Microanalysis (Springer, Berlin, 1984).

${ }^{40} \mathrm{~L}$. Mandel and E. Wolf, Optical Coherence and Quantum Optics (Cambridge University Press, 1995).

${ }^{41}$ J. C. H. Spence and J. M. Zuo, Ultramicroscopy 69, 185 (1997).

${ }^{42}$ H. Lichte, Philos. Trans. Ser. A Math. Phys. Eng. Sci. 360, 897 (2002).

${ }^{43}$ V. D. D. H. Lichte, and J. C. Spence, Ultramicroscopy 81, 187 (2000).

${ }^{44}$ J. Verbeeck, G. Bertoni, and H. Lichte, Ultramicroscopy 111, 887 (2011). 